\title{
Perspectiva Temporal de Estudiantes Universitarios Mexicanos y Colombianos
}

\author{
Time Perspective of University Students Mexican and Colombian
}

\author{
Daniel González Lomelí ${ }^{1}$, María de los Ángeles Maytorena ${ }^{1}$, Lucila Cárdenas-Niño ${ }^{2}$ y César \\ Octavio Tapia-Fonllem ${ }^{1}$
}

\section{Resumen}

El estudio buscó comparar la orientación temporal de estudiantes universitarios colombianos $(\mathrm{N}=770)$ y mexicanos $(\mathrm{N}=495)$ de recién ingreso, los cuales participaron de manera informada y voluntaria. Los resultados reflejan que los estudiantes colombianos, en comparación con los mexicanos, muestran una mayor orientación al presente hedonista, y una mayor orientación hacia el pasado negativo. Las diferencias entre ambos grupos de estudiantes no resultaron significativas en las escalas orientación al futuro, presente fatalista y pasado positivo. Se realizó un análisis factorial confirmatorio por modelamiento de ecuaciones estructurales y se logró construir un modelo formado por cinco variables latentes de primer orden. Los constructos pasado negativo, pasado positivo, presente fatalista y futuro quedaron integrados por cuatro variables manifiestas; mientras que el factor presente hedonista se conformó por cinco variables observadas. Los indicadores de bondad de ajuste prácticos son adecuados.

Palabras clave: perspectiva temporal, estudiantes universitarios, modelamiento estructural, ZTPI, MéxicoColombia

\begin{abstract}
The aim of this study was to compare the temporal orientation of Colombian and Mexican university students who participated in an informed and voluntary way, where 770 new entry students were Colombian and 495 Mexica. The results demonstrate that Colombian students in comparison with Mexican students, show more orientation towards a hedonistic present and towards a negative past. The differences between Colombian and Mexican scales in the orientation to the future, fatalistic present and positive past were not significant at all. A confirmatory factor analysis by structural modeling was performed and a model consisting of five factors was also built. The negative past, positive past, fatalistic present and future factors, are integrated by four indicators while the hedonistic present factor was formed by five indicators. The indicators of good fit are adequate.
\end{abstract}

Keywords: time Perspective, university students, structural modeling, ZTPI, Mexico-Colombia

En México la investigación fue financiada por el CONACyT a través del proyecto de CB-167235 y en Colombia se agradece a la Universidad Pedagógica y Tecnológica de Colombia por la colaboración en el financiamiento de la primera parte de este estudio, mediante convocatoria de la DIN 2009.

\footnotetext{
${ }^{1}$ Departamento de Psicología y Ciencias de la Comunicación, Universidad de Sonora, México.

${ }^{2}$ Departamento de Psicología,Universidad de San Buenaventura - Medellín, Colombia.

Correspondencia: Daniel González Lomelí, Retorno Rancho Bonito \#65, Rancho Bonito Residencial, C.P. 83170, Hermosillo, Sonora, México. Correo: dgonzalez@psicom.uson.mx
} 


\section{Introducción}

En psicología el tiempo es importante visto como una dimensión de la conciencia, es decir, como la forma en que los seres humanos damos orden a nuestra experiencia; prueba de ello es el hecho de que el área de la percepción temporal es una de las más antiguas en la investigación científica psicológica (Galarraga \& Stover, 2016; Roeckelein, 2000, 2008).

Las personas saben que el tiempo es limitado y, para planear su vida, realizan cálculos de su duración. Se planean actividades de todo tipo por lo que el tiempo se convierte en el recurso más preciado y es el único que con certeza se agotará por lo que es función de cada persona administrar y usar ese tiempo como lo considere pertinente y todo parece indicar que las decisiones sobre su uso se basan en la forma que la gente percibe al tiempo (Zimbardo \& Boyd, 2008). De ahí la importancia que adquiere el concepto de perspectiva temporal el cual se refiere a la forma en que las personas dividen el continuo flujo de experiencias en marcos temporales como el pasado, presente y futuro para darle coherencia a su vida. Mucha gente presenta un sesgo hacia alguna de las orientaciones temporales, es decir, unos viven más en el pasado, otros sólo se ocupan del presente y unos más se centran en el futuro lo cual afecta la percepción que tenemos del tiempo (Corral, 2010) y las decisiones correspondientes que se tomen en el presente.

En opinión de Zimbardo y Boyd (1999) la perspectiva temporal es un aspecto de gran importancia para la formación de una persona, puede concebirse también como una dimensión fundamental de la construcción del tiempo psicológico, la cual incluye los procesos cognoscitivos que clasifican la experiencia humana en comportamientos del pasado, presente y futuro. El constructo perspectiva temporal es fundamental para comprender la forma en la que los individuos perciben y utilizan su tiempo. Según Keough, Zimbardo y Boyd (1999) es "un proceso inconsciente por medio del cual el acontecer continuo de los hechos personales y sociales se distribuyen en clases temporales elegidas con el fin de dar orden, coherencia y significado a esos eventos. Tales marcos temporales, pasado, presente y futuro, ayudan a codificar, almacenar y evocar situaciones experimentadas, metas, contingencias y escenarios imaginados" (p. 150).

Zhang, Howelly y Stolarski (2013) se propusieron determinar las relaciones entre tener un balance de la perspectiva del tiempo con varias medidas del bienestar subjetivo, para lo cual seleccionaron varias muestras que en total suman 1739 personas con el fin de probar las relaciones entre las variables de interés. Para medir el balance de la perspectiva del tiempo se hizo uso del Inventario de Perspectiva Temporal de Zimbardo y Boyd. Los resultados refieren que quienes tienen una orientación al pasado positivo $(\mathrm{PP})$, presente hedonista (PF) y futuro (F) reportaron altos niveles de bienestar subjetivo mientras que las personas que se orientan hacia el pasado negativo $(\mathrm{PN})$ y presente fatalista $(\mathrm{PF})$ reportan niveles bajos de bienestar subjetivo.

Oyanadel y Buela-Casal (2014) emplearon el Inventario de Perspectiva Temporal de Zimbardo para comparar a personas con trastornos mentales graves y personas saludables con medidas de orientación y estimación temporal y evaluar si el perfil temporal influye en la calidad de vida de personas con ese tipo de trastornos. El estudio se realizó con un diseño cuasi-experimental en el que participaron 167 personas de un grupo clínico a las que se comparó con un grupo igual de personas saludables con características similares. Dentro de los instrumentos se empleó el inventario de perspectiva temporal de Zimbardo y Boyd, una versión modificada y adaptada para Chile. Se encontró que en los grupos clínicos existe mayor puntuación en Pasado Negativo y en Presente Fatalista, resultado que se atribuye a que la orientación al pasado, negativa, y una actitud pesimista con el presente juegan un importante rol en la comprensión de los trastornos mentales graves.

Otro estudio realizado con una muestra de la población brasileña (Milfont, Andrade, Bello y Pessoa, 2008) indica que el presente hedonista correlaciona positivamente con el uso de alcohol y negativamente con religiosidad, mientras que la orientación al futuro correlaciona positivamente con salud y negativamente en lo concerniente a ser un bebedor; en un estudio transnacional de cinco países sobre tabaquismo se encontró que los no fumadores fueron significativamente más 
propensos a la orientación hacia el futuro (66\%) que los fumadores (57\%) (Sansone et al., 2013).

En España Díaz-Morales (2006) al validar el Inventario de Perspectiva Temporal de Zimbardo (ZTPI) encontró que los jóvenes (19-29 años) obtenían una mayor puntuación en la orientación al presente hedonista, mientras que los adultos de mediana edad (30-40 años) se orientaban más hacia el futuro.

El estudio de la perspectiva temporal ha arrojado evidencia de que la orientación temporal que se dé a nuestra vida, afectan los estilos sustentables (Corral \& Pinheiro, 2006); otras investigaciones señalan también que las orientaciones temporales inciden en el comportamiento de estudio (González, Maytorena, Lohr, \& Carreño, 2006). Mientras que Horstmanshof y Zimitat (2007) llegaron a la conclusión de que la orientación temporal futura emerge como un factor importante como mediador entre el compromiso académico que muestran los estudiantes universitarios de primer año, lo cual conduce a su persistencia para concluir los estudios.

Zimbardo y Boyd (1999) proponen la existencia de cinco perspectivas temporales: a) Pasado negativo, b) Pasado positivo, c) Presente fatalista, d) Presente hedonista y e) Futuro. Sobre el proceso de validez, confirmaron estas cinco dimensiones y explicaron el $36 \%$ de la varianza mediante un análisis de componentes principales, lo cual confirmaron a través de ecuaciones estructurales.

La orientación al pasado negativo refleja personas que se centran en eventos dolorosos, no placenteros, que les ocurrieron o que creen que les ocurrieron (esto último encaja con lo que más interesa en esta medida), las personas con esta orientación tienden a ser ansiosos, apáticos y poco amistosos. Por el contrario, alguien con una orientación al pasado positivo captura eventos o percepciones del pasado, ligadas a situaciones felices, lo cual permite a las personas enfrentar adecuadamente situaciones difíciles en el presente, quienes muestran un sesgo hacia esta orientación temporal suelen ser personas agradecidas, conscientes, creativas, amistosas y no se deprimen (Corral, 2010).

El mismo autor (Corral, op. cit.) señala que una orientación al presente fatalista implica un vivir el ahora a costa de la suerte o de la voluntad de otros. Para las personas que muestran un sesgo hacia esta orientación, la voluntad y el control personal no es importante para determinar su destino; suelen ser personas con baja autoestima, ansiedad, infelicidad, depresión, inconsciencia, irresponsabilidad y poca estabilidad emocional. Mientras que una orientación al presente hedonista se presenta en aquellas personas que tienden a disfrutar el aquí y ahora, sin preocuparse por el pasado o por lo que vendrá. Estos individuos son sensuales, buscan la gratificación en el momento, no les interesan los premios del futuro, se centran en el ahora, son impulsivos, buscadores de sensaciones y de riesgos, felices y despreocupados y aquellos con gran orientación al futuro, son buenos para establecer y lograr metas y planear estrategias para cumplir obligaciones a largo plazo. De igual manera visualizan $\mathrm{y}$ formulan objetivos futuros, que influirán en decisiones y juicios en el presente. Tienden a evitar conductas y situaciones de riesgo. Son conscientes, autocontrolados, organizados, creativos, confiables y responsables. La perspectiva temporal futura es una de las más estudiadas por los interesados en la temática, Nunes (2012) refiere además que la perspectiva temporal es considerada como un sistema multidimensional complejo que integra un componente cognitivo $y$ otro afectivo $y$ motivacional.

Galarraga y Stover (2016) refieren que el ZTPI ha pasado por varios procesos de adaptación en países como Francia, España, Brasil, Portugal, Japón, Chile, entre otros y ha presentado variaciones con relación al número de dimensiones y el número de reactivos pero en todos los casos la varianza explicada es superior a $30 \%$. Estas autoras realizaron un estudio para validar y adaptar el ZTPI en estudiantes argentinos de nivel medio.

En el estudio de Zhang, Howell y Stolaski (2013) antes citado se emplearon los 56 reactivos del Inventario de Perspectiva Temporal pero no especifican haber realizado alguna adaptación previa sin embargo, encontraron ciertas similitudes en las correlaciones en relación con que la perspectiva de presente hedonista es la que más figura en asociación con la felicidad. Mientras que Oyanadel y Buelna-Casal (2014) 
reportan que se mantiene la estructura factorial de la versión original con cinco factores y con confiabilidad que va de .59 a .80 vía alfa de Cronbach. Se indica además la utilidad clínica de la citada versión.

En este sentido, el objetivo de esta investigación fue comparar la orientación temporal de estudiantes universitarios colombianos y mexicanos, con el fin de utilizar posteriormente el ZTPI como medida que brinde información acerca de la perspectiva temporal y buscar relaciones con el desarrollo académico de los estudiantes de ambas nacionalidades.

\section{Método}

\section{Participantes}

La muestra de este estudio quedó conformada por 1265 estudiantes universitarios, a quienes se les garantizó la confidencialidad y el anonimato, 770 colombianos y 495 mexicanos. Son mujeres $50.6 \%$ de los estudiantes colombianos y $53 \%$ de los estudiantes mexicanos.

La muestra mexicana quedó integrada por 495 estudiantes adscritos en primero (57\%) y segundo semestre (43\%) en la Universidad de Sonora, distribuidos en 6 licenciaturas, tales como tecnología electrónica 38 (7.7), ingeniería civil 122 (24.6), medicina 116 (23.4), trabajo social 98 (19.8), economía 78 (15.7) y lingüística 43 (8.7).

Mientras que la muestra colombiana está conformada por 740 estudiantes pertenecientes a la Universidad Pedagógica y Tecnológica de Colombia Seccional Tunja, distribuidos en 17 diferentes licenciaturas: enfermería 35 (4,5\%), psicología 69 (9,0\%), medicina 29 (3,8\%), derecho 34 (4,4\%), Ing. Civil 70 (9,1\%), ingeniería de sistemas y computación 43 (5,6\%), artes plásticas $2(0,3 \%)$, licenciatura en ciencias naturales $67(8,7 \%)$, ciencias sociales $20(2,6 \%)$, en educación física $69(9,0 \%)$, matemáticas 40 (5,2\%), en psicopedagogía 29 (3,8\%), economía $31(4,0 \%)$, administración de empresas $51(6,6 \%)$, biología $65(8,4 \%)$, ingeniería agronómica 62 $(8,1 \%)$ y veterinaria $54(6,6 \%)$. La mayor representatividad en la muestra está dada por el primer semestre con un porcentaje del 58\%, seguido de segundo semestre con un $39 \%$, y con un porcentaje poco representativo se encuentra tercer semestre con 3\% del total de la muestra.

\section{Instrumentos}

El Inventario de Perspectiva Temporal elaborado por Zimbardo y Boyd (1999) consta de 56 reactivos tipo Likert con cinco opciones de respuesta que van de 1 (muy poco aplicable) a 5 (bastante aplicable) mide las escalas de a) Pasado positivo, la cual evalúa una actitud nostálgica y feliz del pasado; ejemplo: "Los lugares familiares de la infancia, sus sonidos y olores frecuentemente me traen muchos recuerdos maravillosos"; b) Pasado negativo, que refleja una visión del pasado pesimista y negativa; ejemplo de reactivo: "Revivo constantemente en mi mente experiencias pasadas dolorosas"; c) Presente hedonista, formado por elementos relacionados con una actitud ante la vida y el tiempo basada en el disfrute de los placeres del momento actual; como por ejemplo: "Yo intento vivir mi vida lo más plenamente posible, un día a la vez"; d) Presente fatalista es entendido como la ausencia de orientación temporal, sin énfasis en el momento presente, sin nostalgia del pasado, ni interés por el futuro; ejemplo de reactivo: "Ya que lo que tiene que pasar de cualquier forma pasará, lo que yo haga no importa" y; e) Futuro entendido como la tendencia a planificar y realizar metas futuras distantes en el tiempo; ejemplo de reactivo: "Cuando quiero conseguir alguna cosa, me propongo metas y evalúo los recursos necesarios con los que cuento, para alcanzar esos objetivos" (Anexo).

\section{Procedimiento}

Se aplicó el Inventario a los estudiantes a quienes se les garantizó la confidencialidad y el anonimato con su autorización informada y previa anuencia de los profesores a cargo en el momento de la aplicación en una sesión que duró aproximadamente 30 minutos, en las instalaciones de ambas universidades. Los protocolos de respuesta fueron administrados a los estudiantes de la muestra por estudiantes de licenciatura en proceso de titulación y entrenados para ello.

Para el caso de la muestra mexicana, el inventario ya había sido validado en su versión al español (Corral, et al., 2003); para el caso de la muestra de estudiantes colombianos se revisaron los ítems a través de juicio de expertos quienes de forma cualitativa valoraron cada uno de los reactivos considerando su pertinencia, suficiencia, 
coherencia y redacción relacionados con la sintaxis, pragmática y semántica. Los tres expertos en las áreas de aprendizaje y evaluación clínica no reportaron ninguna modificación a los reactivos del Inventario.

Posteriormente se realizó una prueba piloto con el propósito de observar si los ítems presentaban alguna dificultad al momento de responder, el estudio se hizo previa autorización de los estudiantes a quienes se les informaron los objetivos del estudio y autorizaron participar; se les indicó que señalaran lo que no les pareciera claro o donde sintieran confusión respecto al lenguaje. De acuerdo a lo reportado por los estudiantes, el cuestionario presenta un funcionamiento adecuado en la prueba piloto por lo que no se produjeron modificaciones a la redacción de los ítems, probablemente esto se deba a un lenguaje y cultura similar en los dos países, más aún, si vemos que se aplicó en un contexto educativo universitario similar ya que la población pertenece a los primeros semestres de universidades públicas y este contexto permite inferir sinónimos culturales. Por último, se realizó el levantamiento de datos con la muestra previamente descrita.

\section{Análisis de datos}

Se realizaron análisis de consistencia interna de las escalas del ZTPI, análisis de comparación de medias (ttest) para probar diferencias entre las muestrasen estudio y un análisis factorial confirmatorio a través del paquete estadístico EQS (Bentler, 2006) para probar un modelo multifactorial con datos de los estudiantes de ambas nacionalidades.

\section{Resultados}

Análisis factorial confirmatorio. La figura 1exhibe el resultado del análisis factorial confirmatorio, por modelamiento de ecuaciones estructurales de las orientaciones temporales que mide el ZTPI, se seleccionó el método LM. El modelo resultantemuestra que se mantiene una estructura de cinco orientaciones temporales: pasado negativo, pasado positivo, presente hedonista, presente fatalista y futuro. Los factores quedaron integrados por cuatro variables manifiestas a excepción del factor presente hedonista que se conforma por cinco variables observadas. Los ítems que se eliminaron debido a que presentaron relaciones no significativas y ello no permitía la bondad de ajuste del modelo construido.

En el factor Pasado Negativo los pesos factoriales van de .480 a .682 ; y este factor covaría en forma directa y negativa con Pasado Positivo (-.161) y Futuro (-.149) y de forma directa y positiva con los factores Presente Hedonista (.484) y Presente Fatalista (.498). El factor Pasado Positivo presenta pesos factoriales desde .477 hasta .580 y covaría en forma directa y positiva con los factores Presente Hedonista (.197) y Futuro (.387) y de manera negativa con el factor Presente Fatalista (-.106); en el caso del factor Presente Hedonista los pesos factoriales de los indicadores que lo integran van de .432 a .588 y correlaciona de forma directa y positiva con el factor Presente Fatalista (.623) y de forma directa y negativa con Futuro (-.244); para el factor Presente Fatalista los pesos factoriales de sus indicadores se ubican entre .496 y .549 , y covarían negativamente con Futuro (-.296). Por último, el factor denominado Futuro posee pesos factoriales de .475 a .666 con las variables observadas que lo integran.

La similitud entre los pesos factoriales de las variables observadas, indican validez de constructo convergente entre las variables manifiestas y las variables latentes que los definen y el hecho de que los valores de las covarianzas entre las variables latentes sean menores que los pesos factoriales, indica que el modelo de medición resultante posee validez de constructo divergente.

Los indicadores de bondad de ajuste prácticos son adecuados, lo cual se manifiesta con sus indicadores que fueron superiores a .90 para el IBBAN, IBBANN e IAC y menor a .05 para el RMSEA con un intervalo de confianza de .030, .038. Los indicadores estadísticos fueron $\chi^{2}=415.80(17 \mathrm{gl}) p=.000$, por lo que se concluye que este modelo de relaciones de perspectivas temporales, como fue medido en la muestra de estudiantes colombianos y mexicanos, a pesar de que no es el modelo óptimo, es similar al modelo saturado en cuanto a poder de explicación. 


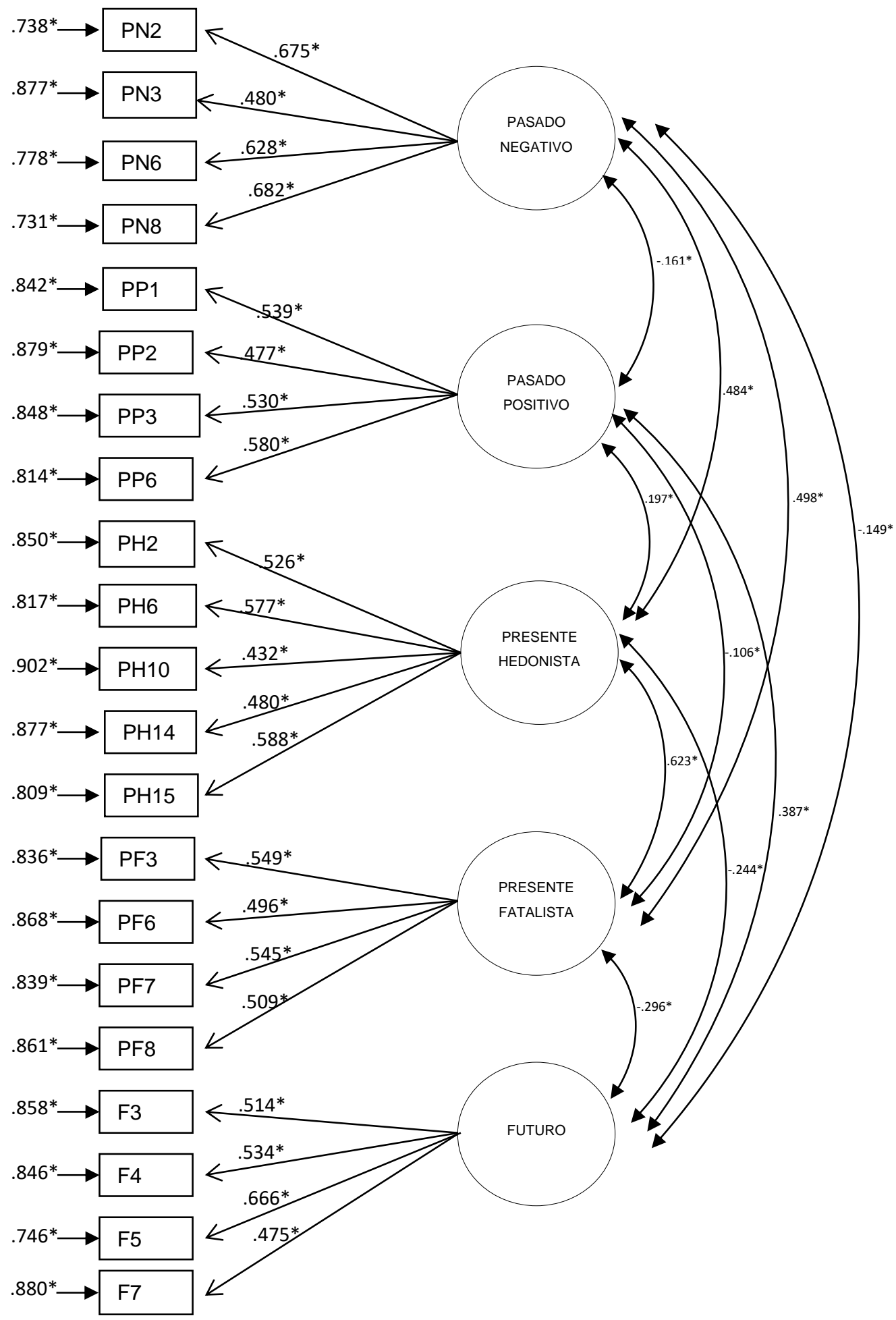

Figura 1. Modelo de perspectiva temporal de estudiantes colombianos y mexicanos. Para cada variable observada $\mathrm{R}^{2}=\left(1\right.$ - error de varianza), $\chi^{2}=415.80(17 \mathrm{gl}) p=.000$. IBAN $=.90 ; \mathrm{IBANN}=.92 ; \mathrm{IAC}=.93$; RMSEA $=.03(.030, .038) . * p<.05 . \mathrm{N}=1265$. 
Tabla 1. Análisis de consistencia interna de las escalas del ZTPI después del AFC

\begin{tabular}{llll}
\hline Escala/reactivo & $M$ & $D E$ & $\alpha$ \\
\hline Pasado negativo & 2.56 & & .71 \\
Revivo constantemente experiencias pasadas dolorosas (PN2) & 2.64 & 1.20 & \\
Yo tuve mi parte de abuso y de rechazo en el pasado (PN3) & 2.45 & 1.21 & \\
Me es difícil olvidar imágenes desagradables de mi juventud (PN6) & 2.66 & 1.19 & \\
Pienso sobre cosas desagradables que acontecieron en el pasado (PN8) & 2.50 & 1.13 & \\
Pasado positivo & 3.63 & & .60 \\
Los lugares familiares de infancia, traen recuerdos maravillosos (PP1) & 3.90 & 1.00 & \\
Me da placer pensar sobre mi pasado (PP2) & 3.06 & 1.12 & \\
En mi pasado existen más cosas buenas que malas para recordar (PP3) & 3.69 & 1.09 & \\
Los recuerdos alegres brotan con facilidad en la mente (PP6) & 3.88 & 0.95 & \\
Presente hedonista & 2.97 & & .65 \\
Hago cosas de manera impulsiva (PH2) & 3.13 & 1.12 & \\
Tomo decisiones al calor del momento (PH6) & 3.08 & 1.12 & \\
Tomar riesgos hace mi vida menos enfadosa (PH10) & 3.11 & 1.12 & \\
Frecuentemente yo sigo a mi corazón más que a mi cabeza (PH14) & 2.85 & 1.14 & \\
Me veo como alguien que se deja llevar por la emoción del momento (PH15) & 2.68 & 1.09 & \\
Presente fatalista & 2.32 & & .61 \\
Ya que lo que tiene que pasar pasará, lo que yo haga no importa (PF3) & 2.42 & 1.14 & \\
La trayectoria de mi vida es controlada por fuerzas... (PF6) & 2.31 & 1.10 & \\
No preocuparse con el futuro, no hay nada que pueda hacer (PF7) & 2.40 & 1.17 & \\
La suerte da más que el trabajo duro (PF8) & 2.15 & 1.07 & \multirow{2}{*}{.63} \\
Futuro & 3.66 & & \\
Cumplir con los plazos por vencer es primero que la diversión (F3) & 3.74 & 0.99 & \\
Me incomoda llegar tarde a mis compromisos (F4) & 3.90 & 1.04 & \\
Cumplo a tiempo mis obligaciones con mis amigos y autoridades (F5) & 3.64 & 0.95 & \\
Termino mis proyectos a tiempo, porque mantengo un constante avance (F7) & 3.38 & 0.98 & \\
\hline & & & \\
& & &
\end{tabular}

Tabla 2. Diferencias de las perspectivas temporales por nacionalidad

\begin{tabular}{lccccc}
\hline Factor & Nacionalidad & $N$ & $M$ & $T$ & sig. \\
\hline Pasado Negativo & Colombia & 770 & 2.720 & 8.358 & .045 \\
& México & 495 & 2.308 & & \\
Pasado Positivo & Colombia & 770 & 3.638 & 0.315 & .164 \\
& México & 495 & 3.625 & & \\
Presente Hedonista & Colombia & 770 & 3.037 & 3.965 & .000 \\
& México & 495 & 2.868 & & \\
Presente Fatalista & Colombia & 770 & 2.453 & 8.053 & .312 \\
& México & 495 & 2.108 & & \\
Futuro & Colombia & 770 & 3.516 & -10.048 & .138 \\
& México & 495 & 3.898 & & \\
\hline
\end{tabular}

Análisis de confiabilidad después del Análisis Factorial Confirmatorio (AFC). Una vez elaborado el análisis factorial confirmatorio, se procedió a realizar el análisis de confiabilidad para cada una de las escalas con los reactivos que las conformaron; las escalas obtuvieron $\alpha$ de .61 a .71. Algunos autores consideran el valor de $\alpha$ de .60 como aceptable (Nieva y Sorra, 2003). Nunnally (1994) también menciona que un alfa de .60 es adecuado para estudios en Ciencias Sociales o en etapas iniciales de la investigación y otros consideran que ese valor es apropiado en estudios de prueba de instrumentos (Wensing, Elwyn, Edwards, Vingerhoets, \& Grol, 2002) como en este caso en particular (Tabla 1).
La Tabla 2 presenta el resultado del análisis de comparación de medias para muestras independientes ( $t$ test) en esos datos se refleja que los estudiantes colombianos puntúan significativamente más alto, tanto en la orientación al presente hedonista, como en la orientación al Pasado Negativo, que los mexicanos. En las orientaciones al Pasado Positivo, Presente Fatalista y Futuro no se encontraron diferencias significativas; pero las puntuaciones indican tendencias de que los estudiantes colombianos son más propensos a las orientaciones de pasado fatalista y pasado positivo que los estudiantes mexicanos que formaron la muestra. 


\section{Discusión}

El análisis psicométrico realizado al Inventario de Perspectiva Temporal de Zimbardo ZTPI (Zimbardo \& Boyd, 1999) con estudiantes universitarios colombianos y mexicanos, en lo que se refiere a la confiabilidad, puede afirmarse que es un instrumento fiable que hace mediciones consistentes, garantizando la estabilidad de las mismas en varias aplicaciones al mismo sujeto. Este resultado se ha podido verificar en otros estudios como el de Oyanadel y Buela-Casal (2014) quienes al comparar a mujeres con trastornos mentales severos y mujeres sanas reportan que se mantiene la estructura factorial de la versión original con cinco factores y con confiabilidad que va de .59 a .80 a través del alfa de Cronbach.

Otro ejemplo se encuentra en Galarraga y Stover (2016) quienes realizaron un estudio para validar y adaptar el ZTPI en estudiantes argentinos de nivel medio. Refieren que el ZTPI ha pasado por varios procesos de adaptación en países como Francia, España, Brasil, Portugal, Japón, Chile, entre otros y ha presentado variaciones con relación al número de dimensiones y el número de reactivos, pero en todos los casos la varianza explicada es superior a $30 \%$.

El modelo estructural resultante confirma la presencia de cinco factores de perspectiva temporal como lo propuso Zimbardo, los cuales se covarían entre sí como teóricamente se esperaba. El hecho de que este inventario posea validez de constructo, tanto convergente como divergente, es un buen hallazgo para futuras investigaciones porque gracias a eso se cuenta con un instrumento que permitirá conocer el perfil de las orientaciones temporales de los estudiantes universitarios, como los que formaron la muestra en este estudio, lo cual da una pauta para seguir buscando relaciones entre la perspectiva temporal y otras variables relacionadas con el aprendizaje (Aguilar et al., 2002; Horstmanshof \& Zimitat, 2007; González et al., 2006) con una medida multidimensional de la perspectiva temporal (Lennings, Burns, \& Cooney, 1998).

Como por ejemplo, el establecimiento de metas académicas por parte de los estudiantes, puede afectar el desempeño académico universitario como lo mencionaron Aguilar, Valencia, Martínez y Vallejo (2002) al mostrar que las medidas de motivación han indicado la necesidad de atender los aspectos motivacionales de los estudiantes, principalmente al ingresar a la universidad tanto en los contenidos curriculares como en las estrategias de evaluación.

En este mismo tenor de las metas académicas Horstmanshof y Zimitat (2007) encontraron que la orientación temporal futura emerge como un factor importante como predictor del compromiso académico que muestran los estudiantes de primer año, lo cual conduce a su persistencia para concluir los estudios. Dado que una carrera universitaria requiere más que sólo asistir a clases (Medrano, Moretti y Ortiz, 2015) estos autores además consideran que la orientación de metas es una de las múltiples variables importantes en el estudio del aprendizaje en universitarios.

En ese sentido, este estudio se une a la breve contribución existente al desarrollo de investigaciones orientadas a la promoción del óptimo desarrollo académico en estudiantes universitariosdado que la perspectiva temporal en su conjunto, es decir, las diferentes orientaciones temporales se han empleado muy poco en el contexto del aprendizaje; a pesar de ello, los resultados obtenidos por González, Maytorena, Lohr y Carreño (2006) y Horstmanshof y Zimitat (2007) demuestran la importancia de investigar sobre ello.

De tal suerte que sí puede ser viable el estudio de la perspectiva temporal en su relación con el desempeño académico, de ahí que se recomienda seguir con esta línea de investigación y estar en mejor posición para atender los procesos motivacionales de los estudiantes al ingresar a la universidad e irlos orientando hacia los propósitos $\mathrm{y}$ metas que tienen para estudiar una carrera universitaria; y la perspectiva temporal puede ser uno de esos procesos. Estas consideraciones se hicieron desde 1998 por Lennings, Burns y Cooney y fueron retomadas por Boniwell, Osin, Linley y Ivanchenko (2010) quienes consideraban que la perspectiva temporal es un constructo cognoscitivo que subyace a la personalidad, al establecimiento de metas, la toma de decisiones e impacto en el bienestar subjetivo.

El hecho de que la escala con mayor puntuación fue la orientación al futuro, indica que 
los estudiantes colombianos y mexicanos, de esta muestra de estudio, tienden a ser personas que pueden establecer y alcanzar metas, planear estrategias y cumplir obligaciones a largo plazo; tienden a evitar conductas y situacionesde riesgo; son personas conscientes, autocontroladas, organizadas, creativas, confiables y responsables (Corral, 2010); contrario a lo que Díaz-Morales (2006) encontró en los jóvenes (19-29 años) españoles, quienes obtuvieron una mayor puntuación en la orientación hacia el presente hedonista y son los adultos (30-40 años) quienes se orientan más hacia el futuro. De tal manera que podría concluirse al respecto que los estudiantes de la muestra de este estudio se encuentran ubicados en lo que quieren hacer con su vida.

La orientación al Pasado Positivo ocupó la segunda posición más alta en puntuaciones, lo cual significa que los estudiantes colombianos y mexicanos que integraron la muestra se caracterizan por ser personas cuyas referencias al pasado, están asociadas a situaciones felices, lo cual les permite enfrentar situaciones difíciles de su vida en el presente.

La puntuación media más baja para ambas muestras fue para la orientación al presente fatalista, dato importante ya que esto indica que los estudiantes de la muestra (tanto colombianos como mexicanos) no presentan tendencia a vivir el ahora a expensas de la suerte o de la voluntad de otros con poco control personal. Las personas con una orientación al presente fatalista tienden a mostrar poca estabilidad emocional (Corral, op. cit.). De tal manera que es buen indicador el que los estudiantes de esta muestra no posean las características antes mencionadas.

Se encontraron diferencias significativas entre estudiantes colombianos y mexicanos en las orientaciones temporales presente hedonista $\mathrm{y}$ pasado negativo, a favor de los estudiantes colombianos; este resultado indica que los estudiantes colombianos, en comparación con los mexicanos, tienden a disfrutar del aquí y el ahora, buscan el disfrute del momento, son impulsivos, buscadores de riesgos, felices y despreocupados (Corral, 2010). También muestran mayor tendencia que los estudiantes mexicanos a centrarse en eventos dolorosos y no placenteros que les ocurrieron, o creen que les ocurrieron por lo que suelen ser ansiosos, desconsiderados, depresivos, apáticos y poco amistosos (op. cit.).

Una limitación del estudio fue que la muestra no fue seleccionada al azar, otra más se debe a que los estudiantes no estaban inscritos en las mismas licenciaturas, ni el número de estudiantes por licenciatura era similar. Lo cual puede ser controlado seleccionando al azar, del listado de licenciaturas que se impartan en ambas instituciones educativas, las licenciaturas de las cuales se obtendrá, de forma aleatoria el número de estudiantes a ser considerados dentro de los grupos de colombianos y mexicanos; buscando que las muestras sean más representativas, a través de un muestreo estratificado por cuotas.

Finalmente, ante la afirmación de Lamas (2008) acerca de que "la acción educativa debe ayudar a los alumnos a ser conscientes de su pensamiento, a ser estratégicos y a dirigir su motivación a metas valiosas" (p.19) se recomienda fomentar en los estudiantes el establecimiento, seguimiento y evaluación de las metas (sociales y personales) durante su formación universitaria, sobre todo las metas académicas.

\section{Referencias}

Aguilar, J., Valencia, A., Martínez, M., \& Vallejo, A. (2002). Un modelo estructural de la motivación intrínseca en estudiantes universitarios. En A. Bazán y A. Arce (Eds.), Estrategias de Evaluación y Medición del Comportamiento (pp. 165-185). Obregón, Sonora, México: ITSON-UADY.

Bentler, P. M. (2006). EQS structural equations program manual. Encino, CA: Multivariate Software.

Boniwell, I., Osin, E., Linley, P.A., \& Ivachenco, G. (2010). A question of balance: Time perspective and well-being in British and Russian samples. The Journal of Positive Psychology, 5(1), 24-40.http://dx.doi.org/10. 1080/17439760903271181

Corral, V., Frías, M., Pavlovich, S., Ibarra, D., Mejía, K., Rolón, L., Valdez, G., \& Velarde, D. (2003). Correlatos de la conducta antisocial y del aprovechamiento escolar en estudiantes de secundaria: Propensión al futuro, uso de la Internet y variables demográficas. 
Investigaciones Educativas en Sonora, 5, 5570.

Corral, V., \& Pinheiro, J. (2006). Sustainability, future orientation and water conservation. European Review of Applied Psychology, 56, 191-198.http://dx.doi.org/10.1016/j.erap.2005 .09 .002

Corral, V. (2010). Psicología de la sustentabilidad. Un análisis de lo que nos hace proecológicos y prosociales. México: Trillas.

Díaz-Morales, J. (2006). Estructura factorial y fiabilidad del Inventario de Perspectiva Temporal de Zimbardo. Psicothema, 18(3), 565-571.

Galarraga, M., \& Stover, J. (2016). Inventario de perspectiva temporal de Zimbardo: Adaptación en estudiantes de nivel medio de Buenos Aires. Psicodebate, 16(1), 109-128. http://dx.doi.org/10.10682/pd.v16i1.540.

González, D., Maytorena, M., Lohr, F., \& Carreño, E. (2006). Perspectiva temporal y morosidad académica en estudiantes universitarios. Revista Colombiana de Psicología, 15, 15-24.

Horstmanshof, L., \& Zimitat, C. (2007). Future time orientation predicts academic engagement among first-year university students. British Journal of Educational Psychology, 77, 703-718. doi:10.1348/00070 9906x160778.

Keough, K., Zimbardo, P., \& Boyd, J. (1999). Who's smoking, drinking, and using drugs? Time perspective as a predictor of substance use.Basic and Applied Social Psychology, 21(2), 149-164.

Lamas, H. (2008). Aprendizaje autorregulado, motivación y rendimiento académico, Liberabit. Revista de Psicología, 14, 15-20.

Lennings, Ch., Burns, A., \& Cooney, G. (1998). Profiles of time perspective and personality: Developmental considerations. The Journal of Psychology, 132(6), 629-641.http://dx.doi.or g/10.1080/00223989809599294.

Medrano, L., Moretti, L., \& Ortiz, A. (2015).Medición del Engagement Académico en Estudiantes Universitarios. Revista Iberoamericana de Diagnóstico y Evaluación - e Avaliação Psicológica, 2(40), 114-123.
Milfont, T., Andrade, P., Belo, R., \& Pessoa, V. (2008). Testing Zimbardo time perspective inventory in a brazilian sample. Revista Interamericana de Psicología/Interamerican Journal of Psychology, 42(1), 49-58.

Nieva, V. F., \& Sorra, J. (2003). Safety culture assessment: a tool for improving patient safety in healthcare organizations. Quality Safe Health Care, 12 (supl), ii17ii23.doi:10.1136/qhc.12.suppl_2.ii17

Nunnally, J. C. (1994). Psychometric Theory, 3aed. New York: McGraw-Hill.

Nunes, I. (2012). O Inventário de Perspectiva Temporal: Estudo de validacao. Revista Iberoamericana de Evaluación - e Avaliação Psicológica,34(1), 117-132.

Oyanadel, C., \& Buela-Casal, G. (2014). Percepción del tiempo y psicopatología: Influencia de la orientación temporal en la calidad de vida de los trastornos mentales graves. Actas Españolas de Psiquiatría, 42(3), 99-107.

Roeckelein, J. E. (2000). The concept of time in psychology: A resource book and annotated bibliography. Wesport: Greenwood Publishing Group.

Roeckelein, J. E. (2008). History of conceptions and accounts of time and early time perception research. En S. Grondin (Ed.), Psychology of Time, (pp. 1-50). Londres: Emerald Group Publishing.

Sansone, G., Fong, G., Hall, P., Guignard, R., Beck, F., Mons, U., Pötschke-Langer,... Jiang, Y. (2013). Time perspective as a predictor of smoking status: findings from the International Tobacco Control (ITC) Surveys in Scothand, France, Germany, China, and Malaysia. BMC Public Health, 13:346. En http://www.biomedcentral.com/1471-2458/1 3/346.doi:10.1186/1471-2458-13-346.

Wensing, M. Elwyn, G., Edwards, A., Vingerhoets, E., \& Grol, R. (2002). Deconstructing patient centred communication and uncovering shared decision making: An observational study. BMC Medical Informatics and Decision Making, 2: 2.doi: 10.1186/1472-6947-2-2

Zhang, J., Howell, R., \& Stolarski, M. (2013). Comparing three methods to measure a balanced time perspective: the relationship 
between a balanced time perspective and subjective well-being. Journal Happiness Studies, 14, 169-184. doi: 10.1007/s10902012-9322-x.

Zimbardo, P., \& Boyd, J. (2008). The Time Paradox: The new psychology of time that will change your life. New York, NY: Simon $\&$ Schuster.

Zimbardo, P., \& Boyd, J. (1999). Putting time in perspective: A valid, reliable individualdifferences metric.Journal of Personality and Social Psychology, 77(6), 1271-1288. 
Anexo

Lea cada oración y responda, de la manera más honesta posible, la pregunta: "La afirmación presentada se aplica a usted, o es verdadera con respecto a usted?" Asígnele una "X" para cada caso, usando los espacios al final de la oración. Por favor, responda a TODAS las preguntas.

\begin{tabular}{|c|c|c|c|c|c|}
\hline & \multicolumn{2}{|c|}{$\begin{array}{l}\text { Muy poco } \\
\text { aplicable }\end{array}$} & Neutro & \multicolumn{2}{|c|}{$\begin{array}{l}\text { Bastante } \\
\text { aplicable }\end{array}$} \\
\hline & 1 & 2 & 3 & 4 & 5 \\
\hline $\begin{array}{l}\text { 1. Participar de una fiesta con amigos es uno de los placeres importantes en la } \\
\text { vida. }\end{array}$ & & & & & \\
\hline $\begin{array}{l}\text { 2. Los lugares familiares de la infancia, sus sonidos y olores frecuentemente } \\
\text { me traen muchos recuerdos maravillosos. }\end{array}$ & & & & & \\
\hline 3. El destino determina mucho de mi vida. & & & & & \\
\hline $\begin{array}{l}\text { 4. Con frecuencia pienso en lo que yo debería haber hecho de manera } \\
\text { diferente en mi vida. }\end{array}$ & & & & & \\
\hline $\begin{array}{l}\text { 5. Mis decisiones son bastante influenciadas por las personas y cosas a mi } \\
\text { alrededor. }\end{array}$ & & & & & \\
\hline 6. Pienso que las personas deberían planear su día cada mañana. & & & & & \\
\hline 7. Me da placer pensar sobre mi pasado. & & & & & \\
\hline 8. Hago cosas de manera impulsiva. & & & & & \\
\hline 9. Si las cosas no se hacen a tiempo, yo no me preocupo por eso. & & & & & \\
\hline $\begin{array}{l}\text { 10. Cuando quiero conseguir alguna cosa, me propongo metas y evalúo los } \\
\text { recursos necesarios con los que cuento, para alcanzar esos objetivos }\end{array}$ & & & & & \\
\hline $\begin{array}{l}\text { 11. En general, en mi pasado existen muchas más cosas buenas que malas } \\
\text { para recordar. }\end{array}$ & & & & & \\
\hline $\begin{array}{l}\text { 12. Cuando escucho mi música favorita, es muy fácil que pierda la noción del } \\
\text { tempo. }\end{array}$ & & & & & \\
\hline $\begin{array}{l}\text { 13. Cumplir con los plazos que están por vencerse y hacer las tareas } \\
\text { necesarias son cosas que vienen primero que la diversión }\end{array}$ & & & & & \\
\hline $\begin{array}{l}\text { 14. Ya que lo que tiene que pasar de cualquier forma pasará, lo que yo haga } \\
\text { no importa. }\end{array}$ & & & & & \\
\hline $\begin{array}{l}\text { 15. Me gustan las historias sobre cómo eran las cosas en los "buenos viejos } \\
\text { tiempos". }\end{array}$ & & & & & \\
\hline 16. Revivo constantemente en mi mente experiencias pasadas dolorosas. & & & & & \\
\hline 17. Yo intento vivir mi vida lo más plenamente posible, un día a la vez. & & & & & \\
\hline 18. Me incomoda llegar tarde a mis compromisos. & & & & & \\
\hline 19. Idealmente, yo viviría cada día como si fuese el último. & & & & & \\
\hline 20. Los recuerdos alegres de buenos tiempos brotan con facilidad en la mente. & & & & & \\
\hline 21. Cumplo a tiempo mis obligaciones con mis amigos y autoridades. & & & & & \\
\hline 22. Yo tuve mi parte de abuso y de rechazo en el pasado. & & & & & \\
\hline 23. Tomo decisiones al calor del momento. & & & & & \\
\hline 24. Yo vivo cada día como se presenta, en lugar de planearlo. & & & & & \\
\hline $\begin{array}{l}\text { 25. El pasado contiene muchos recuerdos desagradables, por eso prefiero no } \\
\text { pensar en él }\end{array}$ & & & & & \\
\hline 26. Es importante ponerle emoción a mi vida. & & & & & \\
\hline 27. Yo cometí errores en el pasado que me gustaría poder borrar. & & & & & \\
\hline $\begin{array}{l}\text { 28. Pienso que es más importante disfrutar de lo que se hace que terminar un } \\
\text { trabajo a tiempo }\end{array}$ & & & & & \\
\hline 29. Me siento nostálgico con respecto a mi infancia. & & & & & \\
\hline $\begin{array}{l}\text { 30. Antes de tomar una decisión, yo evalúo costos y beneficios de esa } \\
\text { decisión. }\end{array}$ & & & & & \\
\hline
\end{tabular}




\begin{tabular}{|c|c|c|c|c|c|}
\hline & $\begin{array}{l}\text { Muy poco } \\
\text { aplicable }\end{array}$ & \multicolumn{2}{|c|}{ neutro } & \multicolumn{2}{|c|}{$\begin{array}{l}\text { Bastante } \\
\text { aplicable }\end{array}$} \\
\hline & 1 & 2 & 3 & 4 & 5 \\
\hline 31. Tomar riesgos hace mi vida menos enfadosa. & & & & & \\
\hline $\begin{array}{l}\text { 32. Para mi es más importante disfrutar el desarrollo de la vida que focalizar } \\
\text { en el punto de destino. }\end{array}$ & & & & & \\
\hline 33. Raramente las cosas resultan como yo esperaba. & & & & & \\
\hline 34. Para mi es difícil olvidar imágenes desagradables de mi juventud. & & & & & \\
\hline $\begin{array}{l}\text { 35. Pensar sobre metas, resultados y productos le quita el placer que siento } \\
\text { al realizar mis actividades. }\end{array}$ & & & & & \\
\hline $\begin{array}{l}\text { 36. Incluso cuando estoy disfrutando el presente, me siento tentado a hacer } \\
\text { comparaciones con experiencias pasadas semejantes. }\end{array}$ & & & & & \\
\hline $\begin{array}{l}\text { 37. Realmente no es posible planear el futuro, porque las cosas cambian } \\
\text { mucho. }\end{array}$ & & & & & \\
\hline $\begin{array}{l}\text { 38. La trayectoria de mi vida es controlada por fuerzas sobre las que yo no } \\
\text { puedo influir. }\end{array}$ & & & & & \\
\hline $\begin{array}{l}\text { 39. No tiene sentido preocuparse con el futuro, ya que a final de cuentas no } \\
\text { hay nada que yo pueda hacer al respecto. }\end{array}$ & & & & & \\
\hline $\begin{array}{l}\text { 40. Termino mis proyectos a tiempo, porque mantengo un constante avance } \\
\text { de las actividades de ese proyecto. }\end{array}$ & & & & & \\
\hline $\begin{array}{l}\text { 41. Generalmente yo me desligo de la conversación cuando mis familiares } \\
\text { hablan sobre cómo eran las cosas en el pasado. }\end{array}$ & & & & & \\
\hline 42. Yo asumo riesgos para ponerle emoción a mi vida. & & & & & \\
\hline 43. Hago listas de las cosas que tengo que hacer. & & & & & \\
\hline 44. Frecuentemente yo sigo a mi corazón más que a mi cabeza. & & & & & \\
\hline $\begin{array}{l}\text { 45. Soy capaz de resistir las tentaciones cuando se que hay trabajo por } \\
\text { hacer. }\end{array}$ & & & & & \\
\hline $\begin{array}{l}\text { 46. Yo me veo a mi mismo como alguien que se deja llevar por la emoción } \\
\text { del momento. }\end{array}$ & & & & & \\
\hline $\begin{array}{l}\text { 47. La vida de hoy es demasiado complicada; prefiero la vida más simple del } \\
\text { pasado. }\end{array}$ & & & & & \\
\hline 48. Prefiero a los amigos que son espontáneos, en lugar de los previsibles. & & & & & \\
\hline $\begin{array}{l}\text { 49. Disfruto los rituales y tradiciones familiares que se repiten con } \\
\text { regularidad. }\end{array}$ & & & & & \\
\hline $\begin{array}{l}\text { 50. Pienso sobre las cosas desagradables que acontecieron conmigo en el } \\
\text { pasado. }\end{array}$ & & & & & \\
\hline $\begin{array}{l}\text { 51. Sigo trabajando en tareas difíciles y no interesantes, si ellas me van a } \\
\text { ayudar a avanzar. }\end{array}$ & & & & & \\
\hline $\begin{array}{l}\text { 52. Gastar hoy en placeres lo que gano es mejor que ahorrar para la } \\
\text { seguridad del mañana. }\end{array}$ & & & & & \\
\hline 53. La suerte da más que el trabajo duro. & & & & & \\
\hline 54. Pienso acerca de las cosas buenas que perdí en mi vida. & & & & & \\
\hline 55. Me gusta que mis relaciones íntimas sean apasionadas. & & & & & \\
\hline 56. Siempre va a haber tiempo para poner al día mi trabajo. & & & & & \\
\hline
\end{tabular}

